\title{
La lectura interfiere con la percepción gustativa: Efecto Stroop con palabras y anagramas
}

\author{
Interference between reading and gustative perception: \\ Stroop effect for words and anagrams
}

\author{
Eugenia Razumiejczyk ${ }^{1}$ \\ Guillermo Macbeth ${ }^{2}$ \\ Universidad Nacional de Entre Ríos, Argentina
}

Resumen. El propósito de este trabajo es evaluar la interferencia intermodal entre la percepción gustativa y las representaciones lingüísticas. Se emplearon estímulos gustativos, palabras y anagramas. Se encontró que los anagramas generaron respuestas más rápidas que las palabras, pero no más acertadas en la identificación de diferentes gustos. Se propone explicar este efecto por el automatismo inferencial evocado para la resolución de los anagramas. Se encontró también que la congruencia entre la información de la lectura y el gusto generó mayor cantidad de aciertos en comparación con las condiciones de incongruencia y control. Se argumenta que este efecto stroop intermodal, entendido como interferencia o facilitación entre modalidades perceptivas, es compatible con una interpretación ecológica.

Palabras clave. Gusto, lectura, palabras, anagramas, stroop.

Abstract. The purpose of this paper is to evaluate the cross-modal interference between taste perception and linguistic representations. Taste stimuli, words and anagrams were employed. Results show that anagrams are processed faster than words, but they are less accurate on taste identification. It is argued that this effect can be interpreted as an automatism; also, findings show that congruence is critical for gustative stimuli recognition. The congruence between gustative and visual modalities generates more recognitions than incongruent and control conditions. An ecological interpretation for this stroop effect is proposed.

Keywords. Taste, reading, words, anagrams, stroop.

\footnotetext{
${ }^{1}$ Eugenia Razumiejczyk, Facultad de Ciencias de la Educacion, Universidad Nacional de Entre Ríos. Dirección postal: Alameda de la Federación 106, E3100GNO, Paraná, Entre Ríos, Argentina. E-mail: eugeniaraz@hotmail.com

${ }^{2}$ Guillermo Macbeth, Facultad de Ciencias de la Educación, Universidad Nacional de Entre Ríos. E-mail: g.macbeth@conicet.gov.ar
} 


\section{Introducción}

La memoria operativa es definida como el sistema de memoria que mantiene y manipula información de manera temporal (Baddeley, 1992). La información percibida del medio a través de los diferentes canales sensoriales tiene una alta probabilidad de estar vinculada. En el proceso de integración perceptual, la memoria operativa permite mantener y procesar la información a pesar de los distintos tiempos en que ésta ingresa al sistema cognitivo (Baddeley, 1992).

Este mecanismo es altamente adaptativo dado que las múltiples fuentes de información provenientes de las distintas modalidades sensoriales se combinan con el objetivo de proporcionar información lo más ajustada posible acerca de las propiedades externas del medio (Driver \& Spence, 2000). En este sentido, se enfatiza aquí que la integración intermodal es más la regla que la excepción en el proceso de la percepción. Así, la integración intermodal es un caso paradigmático de la necesidad de ir más allá de la modularidad (Bertelson, 1999; Calvert, Campbell \& Brammer, 2000; Driver \& Spence, 1998). Sin embargo, la memoria operativa presenta limitaciones al procesar dos fuentes de información de forma simultánea.

Estas limitaciones y capacidades de la memoria operativa fueron estudiadas por Stroop (1935), quien realizó un estudio en el que administraba palabras cuyos significados aludían a colores, estas palabras estaban escritas en tintas iguales o diferentes a las que se refería el significado de las palabras. La consigna requería nombrar el color con el que estaba escrita la palabra en el menor tiempo posible. Los resultados mostraron que cuando los estímulos eran congruentes se registraban mayores aciertos y menores tiempos de reacción (MacLeod, 1991). En oposición, cuando los estímulos eran incongruentes, los participantes evidenciaban significativamente mayor tiempo de reacción en sus respuestas.

La congruencia remite a la coherencia semántica entre el color del estímulo y el significado de la palabra. Resulta así congruente la palabra rojo cuando se presenta escrita como estímulo visual en color rojo.
La incongruencia se operacionaliza mediante una discrepancia, por ejemplo cuando la palabra rojo se escribe en tinta azul.

Se explicaron los resultados de la tarea stroop que estudia fenómenos de la memoria operativa, a partir de la hipótesis de automaticidad que afirma que leer una palabra es un proceso más automático que nombrar el color de la tinta con la que está escrita (Brown, Ross-Gilbert \& Carr, 1995; LaBerge, 1990; Posner \& DeHaene, 1994). En este sentido, cuando los estímulos eran incongruentes, la demanda del procesamiento en la memoria operativa era más ardua y favorecía el proceso automático de la lectura de la palabra por sobre el procesamiento del color de la palabra.

Se enumeraron tres criterios que describieron el procesamiento automático de la palabra en la prueba stroop: 1) el procesamiento siempre se produce cuando se presenta el estímulo apropiado (palabra), independientemente de que exista la intención de procesar el estímulo; 2) el procesamiento de la palabra no está disponible a la conciencia, por lo tanto, no es posible detener el proceso una vez comenzado; y 3) el procesamiento de la palabra no demanda recursos atencionales (Posner \& Snyder, 1975; Schneider \& Shiffrin, 1977; Shiffrin \& Schneider, 1977).

Diversos autores, no obstante, interpretan el fenómeno stroop como un efecto de competencia atencional entre estímulos (Cho, Lien \& Proctor, 2006; Kahneman \& Chajczyk, 1983; Kim, Cho, Yamaguchi \& Proctor, 2008; Mitterer, LaHeij \& Van der Heijden, 2003), esto es, como un proceso de atención selectiva (Lamers \& Roelofs, 2007). En la tarea stroop, la información solicitada se refiere al color de la palabra escrita y el distractor es la palabra como estímulo lingüístico.

Así, la prueba stroop requiere la inhibición de distractores para lograr el procesamiento de la información que solicita la consigna (Kirn, Kirn \& Chun, 2005; Sreenivasan \& Jha, 2007). La interferencia stroop se observa cuando la función ejecutiva de la atención falla por los distractores incongruentes (White $\&$ Prescott, 2007). Se genera de esta manera un mayor 
tiempo de reacción o se cometen mayores errores en las respuestas.

Se han desarrollado diversas modificaciones a la tarea stroop original. Por ejemplo, Regan (1978) administró palabras referidas a colores aunque escritas en color negro pero con la primera letra presentada en color congruente o incongruente con el significado de la palabra. Asimismo, Dyer (1973) realizó un experimento en el que ubicó la palabra escrita en color negro de un lado y una mancha de color del otro lado de un punto de fijación. De una manera similar, Kahneman y Chajczyk (1983) colocaron la palabra por arriba o por debajo de una mancha de color. Estos estudios hallaron interferencias significativas.

El efecto stroop se ha estudiado también en situaciones intermodales (Prescott, Johnstone \& Francis, 2004; Rolls, 2004; White \& Prescott, 2007; Razumiejczyk, Jáuregui \& Macbeth, 2012). En la tarea stroop intermodal se administran de manera simultánea dos estímulos en modalidades diferentes con la consigna de procesar sólo uno, de modo que el otro funcione como distractor. De esta manera, entran en competencia recursos computacionales de la memoria operativa para el procesamiento simultáneo de dos tipos de información presentadas en dos modalidades diferentes.

La información que se presenta en dos modalidades perceptuales distintas, puede ser congruente, incongruente o control. En este sentido, la variable independiente tiene que ver con el tipo de congruencia de la información entre las modalidades. Se intenta medir la interferencia a través de las variables dependientes del tiempo de reacción y el número de aciertos.

En el paradigma stroop, a diferencia del paradigma que estudia el fenómeno de priming, los estímulos se presentan de modo simultáneo. En este último, en cambio, los estímulos ya sean de la misma modalidad o de modalidades diferentes se presentan de forma sucesiva. En el priming se operacionaliza esta presentación secuencial de estímulos mediante el Stimulus Onset Asynchrony (SOA) que es la medida de tiempo transcurrido entre la presentación del prime y el target (Veldhuizen,
Oosterhoff \& Kroeze, 2010).En el paradigma stroop la presentación de estímulos es simultánea, tal como ocurre en el experimento de este artículo.

White y Prescott (2007) estudiaron la interferencia stroop intermodal entre la percepción gustativa y la olfativa; estos autores realizaron un experimento en el que solicitaron a los participantes que identificaran un estímulo gustativo que se administraba junto con un estímulo olfativo. Según la presentación de los pares de estímulos se generaron tres condiciones: 1) congruencia: el estímulo olfativo y el estímulo gustativo pertenecían al mismo alimento; 2) incongruencia: el estímulo olfativo y el gustativo no pertenecían al mismo alimento y; 3) control: el estímulo olfativo era agua. Los resultados mostraron una facilitación en la identificación de los estímulos gustativos en la condición de congruencia. Los autores enfatizaron el significado adaptativo de estos resultados.

Por su parte, Razumiejczyk et al. (2012) han estudiado la interferencia stroop intermodal entre las modalidades gustativa y lingüística auditiva. Administraron a los participantes estímulos gustativos que debían identificar lo más rápidamente posible mientras se presentaban palabras en forma auditiva. La interferencia stroop resultó menor en el nivel de estímulos congruentes, esto es, cuando el estímulo gustativo y la palabra presentada en forma auditiva coincidieron. En oposición, los niveles de estímulos incongruentes y controles generaron tiempos de reacción más lentos y mayor cantidad de errores. Estos resultados son coherentes con lo observado por White y Prescott (2007).

Razumiejczyk, Macbeth y Adrover (2011) compararon la interferencia stroop intermodal entre la percepción gustativa, 1) las representaciones visuales pictóricas, y 2) las representaciones visuales lingüísticas. Los resultados mostraron que las palabras funcionaron como un mayor distractor que las imágenes pictóricas en la tarea stroop intermodal, necesitando un mayor tiempo de procesamiento para la identificación del estímulo gustativo administrado. Los distractores lingüísticos produjeron una mayor competencia atencional con los estímulos gustativos que los 
distractores pictóricos, necesitando un mayor tiempo de reacción y produciendo mayores errores en la identificación de los estímulos gustativos. Se concluyó que el mayor tiempo de procesamiento obtenido en la condición de información lingüística se debió a la mayor complejidad en el procesamiento de este tipo de representaciones mentales de alto nivel.

De este modo, la interferencia stroop intermodal entre el gusto y la visión es mayor con estímulos como palabras que con imágenes visuales debido a que el requerimiento atencional de las primeras es más arduo. En este contexto, se sugiere que el procesamiento habitual de las representaciones visuales lingüísticas forma parte del ajuste ecológico del individuo al ambiente. Se enfatiza aquí que las palabras son estímulos naturales presentes en el medio ambiente y en la vida cotidiana del ser humano. Se sugiere por ello que el procesamiento habitual de las representaciones visuales lingüísticas forma parte de la adaptación ecológica del individuo al ambiente.

Acerca de los anagramas, Foley y Foley (2007) sostienen que existen fáciles y difíciles; estos autores también afirman que los anagramas implican un gasto computacional de la memoria operativa al enfrentarse a la tarea de resolución de problemas. Resulta interesante conocer cómo se comporta la mente frente a la consigna de procesar una fuente de información en la modalidad gustativa cuando la modalidad visual lingüística que la acompaña se encuentra desordenada.

A partir de tal pregunta y sobre la base de los resultados obtenidos en estudios previos (White \& Prescott, 2007; Razumiejczyk et al., 2011, 2012) resulta relevante la comparación de la interferencia stroop intermodal entre la percepción gustativa, 1) las representaciones visuales lingüísticas coherentes (palabras); y 2) las representaciones visuales lingüísticas incoherentes (anagramas). Por ello, el presente estudio se propone dos objetivos específicos: 1) describir la interferencia stroop intermodal entre representaciones gustativas y $i$ ) visuales lingüísticas coherentes (palabras) y ii) visuales lingüísticas incoherentes (anagramas) y; 2) comparar la interferencia stroop intermodal entre las modalidades mencionadas. Con ello se espera conocer la calidad de la interferencia en la memoria operativa entre la percepción gustativa y las representaciones lingüísticas de dos clases, palabras y anagramas.

Las hipótesis principales de este trabajo afirman que el tiempo de procesamiento de los estímulos congruentes es menor que los estímulos incongruentes y controles, tanto en las palabras, como en los anagramas, por un lado, y que, al comparar las palabras (Grupo 1) y los anagramas (Grupo 2), los segundos se procesarán más rápido aunque no se diferencien en el número de aciertos en el nivel de estímulos congruentes. Se justifican estas hipótesis a partir de estudios previos sobre procesos inferenciales que han encontrado que las tareas que evocan automatismos se ejecutan más rápido (Wason \& Johnson-Laird, 1972).

\section{Método}

\section{Participantes}

La muestra total estuvo conformada por 66 estudiantes argentinos universitarios cuya edad promedio fue de 24,03 años ( $D E=6.41$ años). Participaron 45 mujeres $(68.2 \%)$ y 21 varones $(31.8 \%)$. $\mathrm{Al}$ igual que en estudios previos (Razumiejczyk et al., 2011, 2012) se determinaron los siguientes criterios de inclusión de la muestra: 1) tener entre 20 y 40 años de edad (West, 2004); 2) ser no fumador y; 3) no haber ingerido ningún alimento ni bebida que no fuera agua durante las tres horas previas al experimento.

El procedimiento fue llevado a cabo siempre a la misma hora del día. La participación fue libre, voluntaria y con consentimiento informado. Cada participante fue asignado al azar al Grupo $1(n=38)$ o al Grupo $2(n=28)$.

Diseño

Se empleó un diseño experimental mixto de dos grupos aleatorios (Kuehl, 1999) para realizar comparaciones intra-sujetos e inter-sujetos. Se determinó como factor de comparación inter-sujetos la presentación de palabras (Grupo 1) o anagramas (Grupo 2); se definió como factor de comparación intra-sujetos a la congruencia. Para esta variable independiente intra-sujetos se fijaron tres niveles 
según la relación entre el estímulo gustativo y el visual lingüístico: 1) estímulos congruentes: el estímulo gustativo y el visual son congruentes cuando la palabra o el anagrama coinciden con el nombre del estímulo gustativo; 2) estímulos incongruentes: el estímulo gustativo y el estímulo visual son incongruentes cuando la palabra o el anagrama no coinciden con el nombre del estímulo gustativo pero representa una fruta y; 3 ) estímulos controles: el estímulo gustativo y el visual no coinciden pero la palabra o el anagrama no representan un comestible.

Se determinaron como variables dependientes el tiempo de reacción y el número de aciertos. El tiempo de reacción es la cantidad de tiempo medido en segundos con precisión de centésimos entre la presentación de los estímulos y la respuesta verbal del participante. El número de aciertos se operacionalizó como la cantidad de respuestas correctas obtenidas por el participante en la actividad de identificación del estímulo gustativo administrado.

\section{Materiales}

Se administraron como estímulos gustativos durazno, ciruela, frutilla y naranja en forma de papilla licuada a temperatura ambiente. Previamente se realizó un estudio de validación (Razumiejczyk et al., 2010) en el que se seleccionaron estos estímulos de una colección de siete gustos. El coeficiente $\alpha$ de Cronbach para la identificación de estos estímulos resultó de 0.536, lo cual sugiere una homogeneidad psicométrica moderada según Fleiss, Levin y Paik (2004). Para calcular el $\alpha$ de Cronbach se empleó el número de aciertos logrado por cada participante.

Como estímulos visuales se presentaron en la pantalla de una computadora palabras al Grupo 1 y anagramas al Grupo 2, ambas escritas en color negro sobre un fondo blanco. Los anagramas se referían a las mismas palabras pero con las sílabas desordenadas al azar. La Tabla 1 presenta la organización de los estímulos gustativos, las palabras (Grupo 1) y los anagramas (Grupo 2) en función del factor congruencia. La

Tabla 1

Organización de los estímulos gustativos y visuales en función del factor congruencia

\begin{tabular}{llll}
\hline \multicolumn{1}{c}{ Factor Congruencia } & \multicolumn{1}{c}{ Estímulo Gustativo } & \multicolumn{1}{c}{$\begin{array}{c}\text { Palabra } \\
\text { (Grupo 1) }\end{array}$} & $\begin{array}{c}\text { Anagrama } \\
\text { (Grupo 2) }\end{array}$ \\
\hline Estímulos congruentes & Frutilla & FRUTILLA & LLATIFRU \\
Estímulos congruentes & Durazno & DURAZNO & RAZDUNO \\
Estímulos congruentes & Ciruela & CIRUELA & LARUECI \\
Estímulos congruentes & Naranja & NARANJA & JANARAN \\
Estímulos incongruentes & Frutilla & BANANA & NABANA \\
Estímulos incongruentes & Durazno & ANANÁ & NÁANA \\
Estímulos incongruentes & Ciruela & SANDÍA & DÍSANA \\
Estímulos incongruentes & Naranja & MANZANA & NAZAMAN \\
Estímulos controles & Frutilla & MONEDA & NEMODA \\
Estímulos controles & Durazno & ANTEOJOS & JOSANOTE \\
Estímulos controles & Ciruela & BICICLETA & CLECIBITA \\
Estímulos controles & Naranja & RELOJ & LOJRE \\
\hline Nota. Para configurar estos estímulos visuales se utilizó la denominación habitual del español hablado en Argentina por los \\
participantes en el estudio.
\end{tabular}


totalidad de los utensilios (cucharas, vasos y elementos de higiene) se desecharon luego de la utilización por cada participante.

\section{Procedimiento}

Previo a que el experimento tuviera lugar, se llevó cabo la sección del consentimiento informado. El investigador leía a cada participante, de manera individual, el consentimiento y luego lo explicaba verbalmente. Finalmente, el participante lo firmaba e ingresaba al laboratorio en donde lo estaban esperando los experimentadores para comenzar con el procedimiento.

El consentimiento informado consistía en darse por enterado que 1) el propósito general de la investigación era estudiar el comportamiento de algunos fenómenos psicológicos relacionados con la cognición humana; 2) el procedimiento en el que participará no era engañoso; 3) la participación en la investigación no implica ningún riesgo o molestia de ninguna clase; 4) era libre de retirarse de la investigación en cualquier momento sin que eso tuviera consecuencias negativas de ningún tipo; 5) el experimentador informará previo al comienzo del procedimiento cada uno de los pasos que éste implicaba; 6) el investigador responderá a cualquier pregunta respecto de los objetivos del estudio cuando haya concluido la sesión de recolección de datos.

Asimismo, el participante firmaba la aceptación de la publicación de los resultados del estudio teniendo en cuenta que la información a difundir sería anónima, por lo que su identidad se mantendría siempre reservada. Se preguntaba, de forma excluyente, si era alérgico a algún alimento. Si el participante respondía afirmativamente, se le agradecía su buena voluntad y se le explicaba que no podía participar.

El procedimiento era llevado a cabo por dos experimentadores que ignoraban los objetivos del estudio; se utilizó el paradigma de la tarea stroop intermodal (White \& Prescott, 2007). Se administraba a cada participante una serie de estímulos gustativos mientras se presentaba estímulos visuales en la pantalla de una computadora. El experimentador entregaba al participante en la mano una cuchara descartable con 5 mililitros de estímulo gustativo que se llevaba a la boca mientras observaba la pantalla de la computadora en la que simultáneamente se mostraba una palabra. El participante utilizaba un dispositivo que impedía la observación del estímulo gustativo de modo que el participante sólo podía observar las palabras o los anagramas presentados en la pantalla de la computadora durante el experimento.

En este sentido, se evitó el procesamiento incidental del color del estímulo gustativo que se iba a degustar (Morrot, Brochet \& Dubourdieu, 2001); el dispositivo no interfería con el olfato. Se aplicó tal condición debido a la extrema dificultad de identificación del estímulo gustativo que ocurre cuando se bloquean los canales visuales y olfativos según la evidencia aportada en estudios previos (Razumiejczyk, Macbeth \& López Alonso, 2008). La Figura 1 muestra el dispositivo que se diseñó para este fin.

Un grupo recibió palabras (Grupo 1) y el otro grupo recibió anagramas (Grupo 2) de manera simultánea con la administración de los estímulos gustativos. La consigna requería que el participante verbalice el estímulo gustativo que estaba degustando en el menor tiempo posible mientras observaba la computadora. Las respuestas de identificación del participante eran registradas por un experimentador en cada ensayo.

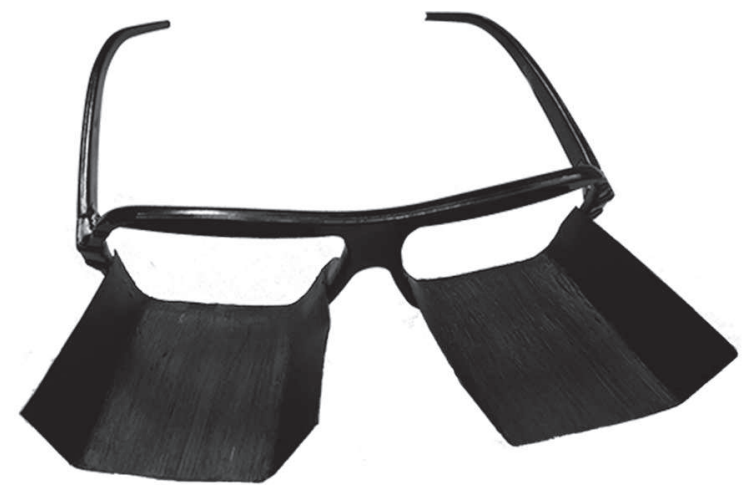

Figura 1. Dispositivo de reducción del campo visual. Dispositivo de bloqueo visual empleado para evitar que la percepción del color interfiera en el reconocimiento del estímulo gustativo durante la lectura de la pantalla de la computadora. 
El tiempo de reacción se registró presionando una tecla de la computadora por parte de cada participante, estos registros se realizaron mediante el programa PsychoPy (Pierce, 2007). Según la relación entre el estímulo gustativo y el estímulo visual lingüístico se determinaron los tres niveles del factor congruencia (estímulos congruentes, incongruentes y controles). Los pares de estímulos (gustativo y visual lingüístico por palabras o por anagramas) fueron administrados a cada participante en un orden aleatorio.

\section{Resultados}

En primer lugar, para poder tratar a la muestra como perteneciente a una misma población, se evaluaron posibles diferencias por género en las variables dependientes, no se hallaron diferencias significativas entre varones y mujeres entre los tres niveles del factor congruencia en las variables tiempo de reacción y número de aciertos, con tamaños del efecto próximos a cero $(d$ Cohen $<.10)$.

En segundo lugar, se analizaron los datos obtenidos en el Grupo 1, que recibió palabras. Para comparar las diferencias del tiempo de reacción entre los tres niveles del factor congruencia se realizó un análisis de varianza intra-sujetos. Se encontró una diferencia estadísticamente significativa entre los estímulos congruentes, incongruentes y

controles $\left(F_{2,35}=13.648, p<.01, \eta_{p}^{2}=.269\right)$. En comparaciones posteriores de a pares se obtuvo que el tiempo de reacción de los estímulos congruentes $(M=12.62, D E=5.7)$ resultó menor $\left(t_{1,36}=-4.459\right.$, $p<.01, d$ Cohen $=.495)$ que el tiempo de reacción de los estímulos incongruentes $(M=15.5, D E=5.92)$. El tiempo de reacción de los estímulos congruentes resultó menor $\left(t_{1,36}=-3.694, p<.01, d\right.$ Cohen $\left.=.366\right)$ que el tiempo de reacción de los estímulos controles $(M=14.73, D E=5.82)$, no se hallaron diferencias significativas entre los estímulos incongruentes y controles $\left(t_{1,36}=1.246, p=.221, d\right.$ Cohen $\left.=.13\right)$.

El número de aciertos logrado por los participantes en los tres niveles del factor congruencia se estudió mediante un análisis de varianza intra-sujetos. Se comprendio una diferencia significativa entre el número de aciertos de los estímulos congruentes, incongruentes y controles $\left(F_{(2,35)}=45.238, p<.01, \eta_{p}^{2}=.55\right)$.

En comparaciones posteriores de a pares se encontró que el número de aciertos de los estímulos congruentes $(M=3.05, D E=0.837)$ resultó mayor $\left(t_{(1,36)}=4.589, p<.01, d\right.$ Cohen $\left.=.954\right)$ que el número de aciertos de los estímulos incongruentes $(M=$ 2.18, $D E=0.982)$. El número de aciertos de los estímulos congruentes resultó mayor $\left(t_{(1,36)}=6.726\right.$, $p<.01, d$ Cohen $=1.015)$, que el número de aciertos de los estímulos controles $(M=2.11, D E=1.008)$. No se encontraron diferencias significativas entre los estímulos incongruentes y los estímulos controles $\left(t_{(1,36)}=-.408, p=.686, d\right.$ Cohen $\left.=.07\right)$.

En tercer lugar, se analizaron los datos obtenidos en el Grupo 2 que recibió anagramas, con el fin de comparar las diferencias del tiempo de reacción entre los tres niveles del factor congruencia se realizó un análisis de varianza intra-sujetos. Se encontró una diferencia estadísticamente significativa entre los estímulos congruentes, incongruentes y controles $\left(F_{(2,25)}=11.575\right.$, $\left.p<.01, \eta_{p}^{2}=.3\right)$.

En comparaciones posteriores de a pares se encontró que el tiempo de reacción de los estímulos congruentes $(M=9.77, D E=4.62)$ resultó menor $\left(t_{(1,}\right.$ ${ }_{26)}=-2.306, p=.029, d$ Cohen $\left.=.277\right)$ que el tiempo de reacción de los estímulos incongruentes $(M=11.22$, $D E=5.75)$ además el tiempo de reacción de los estímulos congruentes resultó menor $\left(t_{(1,26)}=-3.402\right.$, $p<.01, d$ Cohen $=.607)$ que el tiempo de reacción de los estímulos controles $(M=12.83, D E=5.42)$. No se hallaron diferencias significativas entre los estímulos incongruentes y controles $\left(t_{(1,26)}=-1.929, p=.064, d\right.$ Cohen $=.28$ ).

Se comparó el número de aciertos de los anagramas en los tres niveles del factor congruencia a partir del análisis de varianza intra-sujetos; se encontró una diferencia significativa entre el número de aciertos de los estímulos congruentes, incongruentes y controles $\left(F_{2,25}=77.641, p<.01, \eta_{p}^{2}=.3742\right)$. En comparaciones 
Tabla 2

Comparación del tiempo de reacción en los tres niveles del factor congruencia en ambos grupos experimentales

\begin{tabular}{|c|c|c|c|c|c|c|}
\hline & \multicolumn{2}{|c|}{ Estímulos congruentes } & \multicolumn{2}{|c|}{ Estímulos incongruentes } & \multicolumn{2}{|c|}{ Estímulos controles } \\
\hline & Palabras & Anagramas & Palabras & Anagramas & Palabras & Anagramas \\
\hline Media & 12.625 & 9.77 & 15.5 & 11. 224 & 14.736 & 12. 836 \\
\hline$(D E)$ & $(5.7)$ & $(4.624)$ & $(5.923)$ & $(5.755)$ & $(5.822)$ & $(5.427)$ \\
\hline$t_{(1,64)}$ & \multicolumn{2}{|c|}{ 2. 168} & \multicolumn{2}{|c|}{ 2. 933} & \multicolumn{2}{|c|}{1.348} \\
\hline $\mathrm{p}$ & \multicolumn{2}{|c|}{$.034^{*}$} & \multicolumn{2}{|c|}{$<.01 * *$} & \multicolumn{2}{|c|}{.182} \\
\hline d cohen & \multicolumn{2}{|c|}{.54} & \multicolumn{2}{|c|}{0.731} & \multicolumn{2}{|c|}{.336} \\
\hline
\end{tabular}

posteriores de a pares se encontró que el número de aciertos de los estímulos congruentes $(M=2.89, \mathrm{DE}$ $=0.786)$ resultó mayor $\left(t_{1,26}=6.6, p<.01, d\right.$ Cohen $=1.902)$ que el número de aciertos de los estímulos incongruentes $(M=1.32, D E=0.863)$. El número de aciertos de los estímulos congruentes resultó mayor $\left(t_{1,26}=8.811, p<.01, d\right.$ Cohen $\left.=2.084\right)$ que el número de aciertos de los estímulos controles $(M=1.36, \mathrm{DE}$ $=0.678)$. No se hallaron diferencias significativas entre los estímulos incongruentes y controles $\left(t_{1,26}=\right.$ $-.214, p=.832, d$ Cohen $=.252$ ).

Finalmente, se compararon los resultados obtenidos en las variables dependientes en ambos grupos experimentales en los tres niveles del factor congruencia. Se efectuaron las respectivas pruebas $t$ para muestras independientes con el fin de comparar los tiempos de reacción en los tres niveles del factor congruencia en ambos grupos. La Tabla 2 presenta un resumen de los resultados obtenidos.

La Figura 2 presenta un gráfico de barras que compara los tiempos de reacción de los estímulos congruentes, incongruentes y controles en ambos grupos experimentales. Se efectuaron las respectivas pruebas $\mathrm{t}$ para muestras independientes con el fin de comparar el número de aciertos en los tres niveles del factor congruencia en ambos grupos experimentales. La Tabla 3 presenta los resultados obtenidos.

La Figura 3 presenta un gráfico de barras que compara el número de aciertos de los estímulos congruentes, incongruentes y controles en ambos grupos.
Todas las variables comparadas resultaron normales y homocedásticas por las pruebas de KolmogorovSmirnov y Levene respectivamente, por lo cual se aplicaron pruebas paramétricas para contrastar las hipótesis dentro del modelo lineal.

\section{Discusión}

Se ha estudiado la interferencia stroop intermodal entre la percepción gustativa y 1) las representaciones

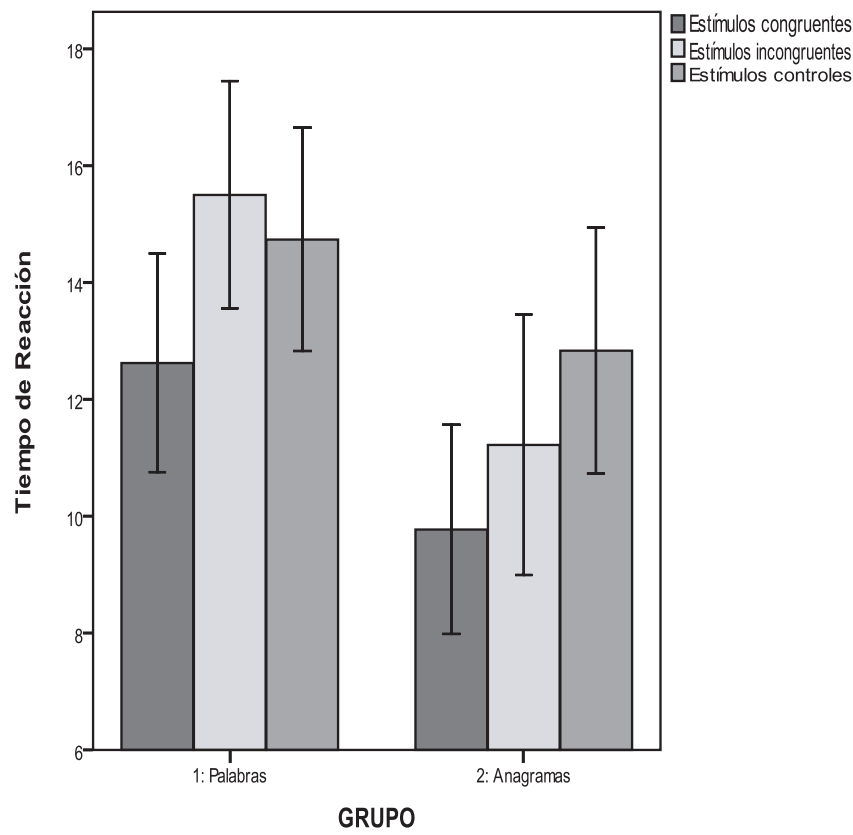

Figura 2. Latencia para palabras y anagramas. Las barras representan medias y las barras de error indican un intervalo. de confianza del 95\% para la media. 
Tabla 3

Comparación del numero de aciertos en los tres niveles del factor congruencia en ambos grupos experimentales

\begin{tabular}{|c|c|c|c|c|c|c|}
\hline & \multicolumn{2}{|c|}{ Estímulos congruentes } & \multicolumn{2}{|c|}{ Estímulos incongruentes } & \multicolumn{2}{|c|}{ Estímulos controles } \\
\hline & Palabras & Anagramas & Palabras & Anagramas & Palabras & Anagramas \\
\hline Media & 3.05 & 2.89 & 2.18 & 1.32 & 2.11 & 1.36 \\
\hline$(D E)$ & $(0.837)$ & $(0.786)$ & $(0.982)$ & $(0.863)$ & $(1.008)$ & $(0.678)$ \\
\hline$t_{(1,64)}$ & \multicolumn{2}{|c|}{.787} & \multicolumn{2}{|r|}{3.709} & \multicolumn{2}{|r|}{3.398} \\
\hline$p$ & \multicolumn{2}{|c|}{.434} & \multicolumn{2}{|r|}{$<.01 *$} & \multicolumn{2}{|r|}{$<.01^{*}$} \\
\hline$d$ cohen & \multicolumn{2}{|c|}{.196} & \multicolumn{2}{|r|}{.921} & \multicolumn{2}{|r|}{.815} \\
\hline
\end{tabular}

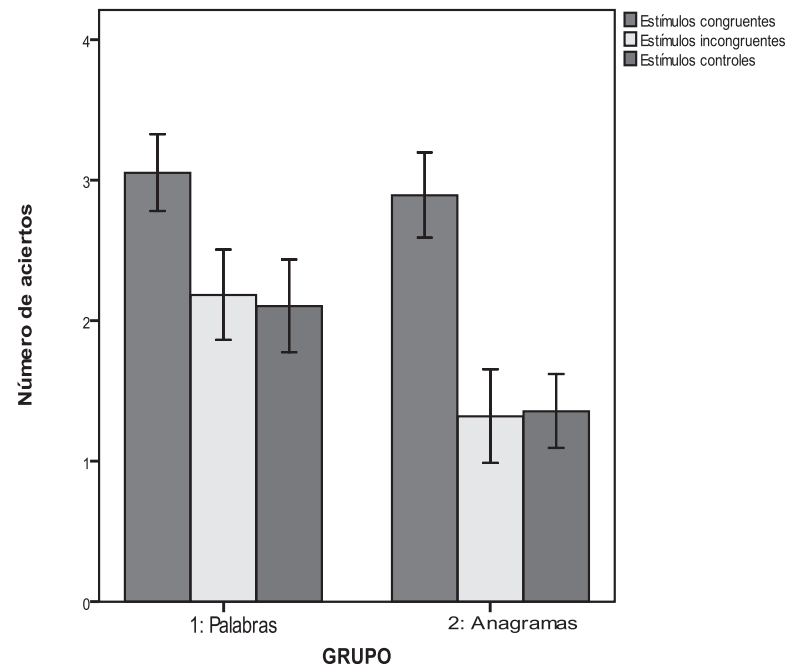

Figura 3. Número de aciertos para palabras y anagramas. Las barras representan medias y las barras de error indican un intervalo de confianza de $95 \%$ para la media.

lingüísticas coherentes, es decir, palabras (Grupo 1), y 2) las representaciones lingüísticas incoherentes, es decir, anagramas (Grupo 2), y se han comparado los resultados de ambos grupos experimentales. La interferencia hallada entre la lectura de palabras o anagramas y la identificación de estímulos gustativos resultó coherente con estudios previos (Stroop, 1935; White \& Prescott, 2007).

Con respecto al Grupo 1 (interferencia entre la percepción gustativa y palabras), los resultados muestran que la interferencia stroop intermodal resultó menor en el nivel de estímulos congruentes, produciendo mayores aciertos y menores tiempos de reacción. Cuando el estímulo gustativo y la palabra se referían al mismo objeto, los participantes respondieron más rápido y de modo más eficaz.

Así, en el nivel de estímulos congruentes la competencia atencional (Cho et al., 2006; Kahneman \& Chajczyk, 1983; Kim et al., 2008; Mitterer et al., 2003) entre los estímulos gustativos y visuales lingüísticos resultó mínima. Esto se debe posiblemente a que la información converge en el mismo objeto que el participante debe mencionar al experimentador. Este resultado extiende los hallazgos de White y Prescott (2007) y Razumiejczyk et al. (2011).

Por otra parte, en el nivel de estímulos incongruentes y controles, los resultados muestran un mayor tiempo de reacción y un menor número de aciertos, es decir, la interferencia stroop intermodal resultó mayor. En este sentido, los participantes no lograron inhibir los distractores visuales lingüísticos para obtener las respuestas relativas a la identificación de los estímulos gustativos de forma rápida y acertada.

Se obtuvieron resultados similares en el Grupo 2 (interferencia entre la percepción gustativa y anagramas), los resultados mostraron que la interferencia stroop intermodal resultó menor en el nivel de estímulos congruentes en comparación con los niveles de estímulos incongruentes y controles.

Esta evidencia es compatible con la interpretación del efecto stroop como proceso de atención selectiva (Cho et al., 2006; Kahneman \& Chajczyk, 1983; Kim et 
al., 2008; Mitterer et al., 2003) dado que los estímulos gustativos compiten por los recursos atencionales con los estímulos visuales. Este hallazgo muestra la adaptación del individuo a los estímulos lingüísticos.

Se propone aquí que este ajuste surge de la necesidad espontánea de procesar la información lingüística, aún sin que tal tarea sea consignada. En el experimento se administraba al participante un estímulo gustativo mientras que se le presentaba en la pantalla de la computadora un anagrama; la consigna requería identificar el estímulo gustativo mientras se observaba la pantalla de la computadora aunque no se daban pistas de que la serie de letras formaba una palabra al ordenar nuevamente las sílabas. Sin embargo, los anagramas funcionaron como distractores compitiendo por los recursos atencionales en la tarea stroop de manera desigual.

Los anagramas incongruentes produjeron mayores errores y mayor tiempo de reacción, mientras que los anagramas congruentes fueron facilitadores en la identificación de los estímulos gustativos. En el nivel de estímulos incongruentes y controles los resultados sugieren que los sujetos no fueron capaces de inhibir esos distractores lingüísticos para cumplir con la tarea de identificar el estímulo gustativo administrado, en coherencia con los hallazgos de White y Prescott (2007) y Razumiejczyk et al. (2011, 2012).
Se enfatiza, en líneas generales, que ambos grupos experimentales presentaron el mismo patrón de resultados frente a las variables dependientes. Se observó una mayor interferencia stroop intermodal en los niveles de estímulos incongruentes y controles con distractores como palabras y anagramas en las variables de tiempo de reacción y número de aciertos. En oposición, el nivel de estímulos congruentes fue facilitador de la identificación de estímulos gustativos tanto con palabras como con anagramas en ambas variables dependientes.

Se propone considerar que el efecto stroop hallado es un fenómeno robusto por producirse tanto con palabras como con anagramas, y replicar hallazgos previos mediante la manipulación del factor congruencia con estímulos gustativos. Se han comparado los resultados obtenidos entre el Grupo 1 y el Grupo 2. Se presenta a continuación la Tabla 4 que resume las comparaciones realizadas.

En el nivel de estímulos congruentes, los resultados mostraron un mayor tiempo de reacción en el Grupo 1 que en el Grupo 2, mientras que el número de aciertos no se diferenció significativamente. En este sentido, las palabras funcionaron como un mejor distractor que los anagramas necesitando un mayor tiempo de procesamiento para la respuesta de identificación del estímulo gustativo, aunque los aciertos fueron similares en ambos grupos experimentales.

Tabla 4

Comparación de las variables dependientes entre el Grupo 1 y el Grupo 2

\begin{tabular}{lll}
\hline Factor Congruencia & Grupo 1 (palabras) & Grupo 2 (anagramas) \\
\hline Estímulos congruentes & Mayor tiempo de reacción & Menor tiempo de reacción \\
& Similar número de aciertos & Similar número de aciertos \\
Estímulos incongruentes & Mayor tiempo de reacción & Menor tiempo de reacción \\
& Mayor número de aciertos & Menor número de aciertos \\
Estímulos controles & Similar tiempo de reacción & Similar tiempo de reacción \\
& Mayor número de aciertos & Menor número de aciertos \\
\hline
\end{tabular}


En el nivel de estímulos incongruentes, los resultados mostraron un mayor tiempo de procesamiento en el Grupo 1 que en el Grupo 2, aunque el número de aciertos fue menor en el Grupo 2 que en el Grupo 1. De esta manera, los participantes identificaron el estímulo gustativo de modo más rápido cuando el distractor era un anagrama que cuando era una palabra, pero acertaron más con palabras que con anagramas, esto es, los anagramas generaron mayor velocidad pero peor identificación de los estímulos gustativos.

En el nivel de estímulos controles, los resultados mostraron que entre el Grupo 1 y el Grupo 2 no hubo diferencias significativas en el tiempo de procesamiento, mientras que el número de aciertos resultó mayor en el Grupo 1 que en el Grupo 2. El tiempo de reacción resultó similar entre los distractores lingüísticos por palabra y por anagrama aunque las palabras generaron mayor cantidad de aciertos en la identificación de los estímulos gustativos. Los anagramas, aunque no se procesaron más rápido, produjeron mayores errores en las respuestas.

En el presente estudio se administraron anagramas sencillos en los que se alteraba el orden de las sílabas de la palabra original, no se aleatorizaron letras sino sílabas. El motivo de esta decisión experimental fue que los anagramas por letras son posiblemente más difíciles de identificar que los anagramas por sílabas, tal dificultad podría perjudicar la captación experimental de la interferencia stroop intermodal buscada. En lugar de generar el fenómeno stroop, se podría generar un fenómeno de resolución de problemas (Foley \& Foley, 2007). De este modo, al utilizar anagramas más accesibles (Foley \& Foley, 2007) se contribuyó a disminuir el tiempo de reacción en comparación con el procesamiento de las palabras.

Esta tendencia se observó en el nivel de estímulos congruentes e incongruentes, mientras que el nivel de estímulos controles no se diferenció en el tiempo de procesamiento. En este sentido, los sujetos tendieron a ordenar espontáneamente la sucesión de sílabas desordenadas. Los sujetos no fueron capaces de evitar el análisis perceptivo de atributos irrelevantes para la tarea (Kahneman, 1997). Esta tendencia inferencial de la mente humana ha sido ampliamente registrada en numerosos estudios sobre razonamiento (Johnson-Laird, 2008).

En sentido opuesto, los datos mostraron un mayor número de aciertos en el Grupo 1, en los niveles de estímulos incongruentes y controles, mientras que no se diferenciaron en el nivel de estímulos congruentes con el Grupo 2. Se observó una mayor cantidad de errores cuando se presentaban anagramas en las condiciones de estímulos incongruentes y controles.

Estos resultados tienen importancia para el enfoque ecológico dado que se generó mayor adaptación cuando los materiales intermodales replicaban condiciones naturales. Es decir, es más frecuente encontrar congruencia que incongruencia en la interacción habitual del ser humano con su entorno. $\mathrm{Al}$ comer frutillas, por ejemplo, es natural que su color sea el rojo y que su gusto sea el de las frutillas y no el de los duraznos. Es razonable por ello que al replicar experimentalmente tales condiciones se registre mayor cantidad de aciertos y mayor velocidad de respuesta.

La condición de incongruencia, en cambio, al ser discrepante del entorno habitual de los participantes generó mayor cantidad de errores y procesamientos más lentos. De la misma manera, tiene sentido encontrar palabras escritas con las sílabas ordenadas en entornos naturales. Las palabras que son leídas habitualmente no tienen la forma de anagramas con sílabas desordenadas al azar, sino que se encuentran ordenadas.

En estudios clásicos sobre procesos inferenciales se ha encontrado que las tareas que evocan automatismos se ejecutan más rápido (Wason \& Johnson-Laird, 1972); en el presente experimento se encontró evidencia compatible con tales estudios.

Se propone aquí que la lectura de anagramas generó automatismos inferenciales que no fueron necesarios para la comprensión de palabras con sílabas ordenadas. Este efecto explicaría la mayor velocidad hallada en el Grupo 2, expuesto a la lectura de anagramas. En las tres condiciones del factor congruencia se hallaron respuestas más rápidas para el Grupo 2. En la condición congruente, si bien no se alcanzó significación estadística para la diferencia 
de velocidad, el tamaño del efecto obtuvo una $d$ de Cohen de 0,336, es decir, entre pequeña y mediana; las otras dos condiciones obtuvieron significación estadística. Los aciertos logrados en la identificación, sin embargo, no mostraron el mismo patrón que la velocidad de respuesta. Se lograron mayores identificaciones gustativas para las palabras que para los anagramas, sin importar que éstos últimos sean procesados más rápido.

En la condición incongruente y en la condición control se hallaron más aciertos para palabras que para anagramas, esto posiblemente se debe a que las palabras exigen menor gasto computacional que los anagramas. Al requerir menos procesamiento, la exposición al error resultó menor y los aciertos resultaron más frecuentes. En la condición congruente, sin embargo, no se hallaron diferencias de aciertos entre palabras y anagramas. Es decir, que los anagramas se procesaron más rápido, pero no mejor sino de modo similar en la condición de congruencia. Este efecto podría entenderse como evidencia compatible con el enfoque ecológico referido anteriormente. Según esta teoría, la réplica experimental de materiales o procesos naturales genera mejores rendimientos por la práctica espontánea acumulada por los participantes en su entorno habitual.

En el presente experimento se encontró, en tal sentido, que las palabras y los anagramas no difieren en condición de congruencia. Es decir, el ajuste natural entre el estímulo gustativo y la lectura de palabras o anagramas resultó indistinto. En este sentido, resulta esperable que las palabras y los anagramas no se diferencien justamente en la condición de congruencia debido a que ésta replica la condición natural del nicho ecológico en el que se desenvuelve el individuo.

En síntesis, el principal aporte de este artículo se refiere a que los patrones de respuestas encontrados entre el Grupo 1 (palabras) y el Grupo 2 (anagramas) fueron similares, pues, los estímulos congruentes se procesaron más rápido y tuvieron mayores aciertos que los incongruentes y controles. Por otra parte, al comparar ambos grupos experimentales en la condición de congruencia los resultados mostraron que el tiempo de reacción resultó menor en el Grupo 2 (anagramas) que en el Grupo 1 (palabras) aunque no se encontró diferencia en el número de aciertos. De esta manera, frente a un anagrama congruente con el estímulo gustativo que el participante estaba degustando, éste identificaba más rápido el estímulo gustativo que frente a una palabra congruente. Se interpretan estos resultados a partir de la tendencia inferencial de la mente humana (Johnson-Laird, 2008).

Una limitación del presente estudio consiste en la cantidad reducida de estímulos gustativos empleados, se recomienda validar nuevos estímulos para extender los hallazgos. Se limitan también los alcances de este estudio al caso particular de anagramas por sílabas. Los anagramas por aleatorización de letras posiblemente resulten demasiado difíciles para generar la interferencia stroop con la modalidad gustativa. En el presente estudio se optó por sílabas en lugar de letras para evitar que la tarea experimental se transforme en una resolución de anagramas en lugar de la identificación gustativa. Sin embargo, es recomendable explorar tal condición en futuros estudios. Se destaca el interés ecológico de este estudio por el empleo de estímulos gustativos naturales que forman parte de la dieta habitual de los participantes, a diferencia de estudios previos que emplearon esencias artificiales o estimulación eléctrica sobre la lengua (Halpern, 2005; Keast, Canty \& Breslin, 2004; Kelling \& Halpern, 1987; Kobayakawa, Ogawa, Kaneda, Ayabe-Kanamura \& Saito, 1999).

\section{Referencias}

Baddeley, A. (1992). Working memory: the interface between memory and cognition. Journal of Cognitive Neuroscience, 4(3), 281-288.

Bertelson, P. (1999). Ventriloquism: a case of crossmodal perceptual grouping. En G. Ashersleben, T. Bachmann \& J. Müsseler (Eds.) Cognitive Contributions to the Perception of Spatial and Temporal Events (pp. 347-362). Amsterdam: Elsevier Science.

Brown, T. L., Ross-Gilbert, L., \& Carr, T. H. (1995). Automaticity and word perception: evidence from stroop and stroop dilution effects. Journal of 
Experimental Psychology: Learning, Memory, \& Cognition, 21, 1395-1411.

Calvert, G. A., Campbell, R. \& Brammer, M. J. (2000). Evidence from functional magnetic resonance imaging of crossmodal binding in the human heteromodal cortex. Current Biology, 10, 649-657.

Cho, Y. S., Lien, M. C. \& Proctor, R. W. (2006). Stroop dilution depends on the nature of the color carrier but not on its location. Journal of Experimental Psychology: Human Perception \& Performance, 32, 826-839.

Driver, J. \& Spence, C. (1998). Attention and the crossmodal construction of space. Trends in Cognitive Science, 2, 254-262.

Driver, J. \& Spence, C. (2000). Multisensory perception: Beyond modularity and convergence. Current Biology, 10, 731-735.

Dyer, F.N. (1973). Interference and facilitation for color naming with separate bilateral presentations of the word and color. Journal of Experimental Psychology, 99, 314-317.

Fleiss, J. L., Levin B. \& Paik, M. C. (2004). Statistical methods for rates and proportions. New York: Wiley.

Foley, M. A. \& Foley, H. J. (2007). Source-monitoring Judgments About Anagrams and Their Solutions: Evidence for the Role of Cognitive Operations Information in Memory. Memory \& Cognition, 35(2), 211-221.

Halpern, B. P. (2005). Temporal characteristics of human taste judgements as calibrations for gustatory event-related potentials and gustatory magnetoencephalographs. Chemical Senses, 30, 228-234.

Johnson-Laird, P. N. (2008). How We Reason. Oxford, UK: Oxford University Press.

Kahneman, D. (1997). Atención y esfuerzo. España: Biblioteca Nueva.
Kahneman, D. \& Chajczyk, D. (1983). Tests of the automaticity of reading: dilution of stroop effects by color-irrelevant stimuli. Journal of Experimental Psychology: Human Perception \& Performance, 9, 497-509.

Keast, R. S. J., Canty, T. M. \& Breslin, P. A. S. (2004). Oral zinc sulphate solutions inhibit sweet taste perception. Chemical Senses, 26, 513-521.

Kelling, S. T. \& Halpern, B. P. (1987). Taste judgements and gustatory stimulus duration: simple taste reaction times. Chemical Senses, 12, 543-562.

Kim, H., Cho, Y. S., Yamaguchi, M. \& Proctor, R. W. (2008). Influence of color availability on the stroop color-naming effect. Perception \& Psychophysics, 70, 1540-1551.

Kirn, S. Y., Kirn, M. S. \& Chun, M. M. (2005). Concurrent working memory load can reduce distraction. Proccedings of National Academy of Sciences of the United States of America, 102, 16524-16529.

Kobayakawa, T., Ogawa, H., Kaneda, H., AyabeKanamura, S. \& Saito, S. (1999). Spatio-temporal analysis of cortical activity evoked by gustatory stimulation in humans. Chemical Senses, 24, 201209.

Kuehl, R. O. (1999). Design of Experiments. Statistical Principles of Research Design and Analysis. Pacific Grove, CA: Duxbury Press.

LaBerge, D. L. (1990). Attention. Psychological Science, 1,156-161.

Lamers, M. J. M. \& Roelofs, A. (2007). Role of gestalt grouping in selective attention: evidence from the stroop task. Perception \& Psychophysics, 69, 13051314.

MacLeod, C. M. (1991). Half a century of research on the stroop effect: An interactive review. Psychological Bulletin, 109 (2), 163-203.

Mitterer, H., La Heij, W. \& Van der Heijden, A. H. C. (2003). Stroop dilution but not word- processing dilution: evidence for attention capture. Psychological Research, 67, 30-42. 
Morrot, G., Brochet, F. \& Dubourdieu. D, (2001). The color of odors. Brain Language, 79, 309-320.

Pierce, J.W. (2007). PsycoPy - Psychophysics software in Python. Journal of Neuroscience Methods, 162, 8-13.

Prescott, J., Johnstone, V. \& Francis, J. (2004). Odortaste interactions: effects of attentional strategies during exposure. Chemical Senses, 29, 331-340.

Posner, M. I. \& DeHaene, S. (1994). Attentional networks. Trends in Neurosciences, 17, 75-79.

Posner, M. I. \& Snyder, C. R. R. (1975). Attention and cognitive control. En R.L. Solso (Ed.) Information processing and cognition: the Loyola symposium. (pp. 55-85). Hillsdale, NJ: Erlbaum.

Razumiejczyk, E. Macbeth, G. \& López Alonso, A. O. (2008). La vinculación entre las modalidades gustativa y olfativa en el reconocimiento del sabor. Psico Logos, 17, 5-12.

Razumiejczyk, E., Bacci, C., Iriarte, M.P., Britos, P., Genovese, I., Grigera Monteagudo, D., Caselli, G. \& Bellucci, P. (2010). Selección de estímulos para el estudio de los procesos cognitivos relacionados con la modalidad gustativa. Psicología y Psicopedagogía, 23.

Razumiejczyk, E., Jáuregui, M. \& Macbeth, G. (2012). Interferencia stroop intermodal entre representaciones gustativas y auditivas. Revista CES Psicología, 5(2), 25-41.

Razumiejczyk, E., Macbeth, G. \& Adrover, J. F. (2011). Comparación de la interferencia stroop intermodal entre representaciones gustativas y visuales por imágenes y por palabras. Boletín de Psicología, 101, 7-20.

Regan, J. E. (1978). Involuntary automatic processing in color- naming tasks. Perception \& Pychophysics, 24, 130-136.
Rolls, E. T. (2004). Multisensory neuronal convergence of taste, somatonsensory, visual, olfactory, and auditory inputs. En G. A. Calvert, C. Spence \& B. E. Stein (Eds.) The Handbook of Multisensory Processes. (pp. 311-331). Cambridge, MA.: MIT Press.

Schneider, W. \& Shiffrin, R. M. (1977). Controlled and automatic human information processing: I. Detection, search and attention. Psychological Review, 84, 1-66.

Shiffrin, R. M., \& Schneider, W. (1977). Controlled and automatic human information processing: II. Perceptual learning, automatic attending and a general theory. Psychological Review, 84, 127-190.

Sreenivasan, K. K. \& Jha, A. P. (2007). Selective attention supports working memory maintenance by modulating perceptual processing of distractkmjiors. Journal of Cognitive Neuroscience, 19, 32-41.

Stroop, J. R. (1935). Studies of interference in serial verbal reactions. Journal of Experimental Psychology, 8, 643-666.

Veldhuizen, M. G., Oosterhoff, A. F. \& Kroeze, J. H. A. (2010). Flavors prime processing of affectively congruent food words and non-food words. Appetite, 54, 71-76.

Wason, P. C. \& Johnson-Laird, P. N. (1972). Psychology of Reasoning. Structure and Content. Cambridge, MA: Harvard University Press.

West, R. (2004). The effects of aging on controlled attention and conflict processing in the stroop task. Journal of Cognitive Neuroscience, 16, 103-113.

White, T. L. \& Prescott, J. (2007). Chemosensory crossmodal stroop effects: congruent odors facilitate taste identification. Chemical Senses, 32, 337-341.

Recibido: 14 de agosto 2013

Aceptado: 25 de febrero 2014 